\title{
Structure of Population Activity in Primary Motor Cortex for Single Finger Flexion and Extension
}

\author{
${ }^{D}$ Spencer A. Arbuckle, ${ }^{1}$ Jeff Weiler, ${ }^{1}$ Eric A. Kirk, ${ }^{2}$ Charles L. Rice, ${ }^{2,3}{ }^{\circ}$ Marc Schieber, ${ }^{4}$ \\ ${ }^{\circledR}$ J. Andrew Pruszynski, ${ }^{1,5,6}$ Naveed Ejaz, ${ }^{1}$ and ${ }^{\circledR}$ Jörn Diedrichsen ${ }^{1,7}$ \\ ${ }^{1}$ Brain and Mind Institute, Western University, London, Ontario N6A3K7, Canada, ${ }^{2}$ School of Kinesiology, Faculty of Health Sciences, Western \\ University, London, Ontario N6A3K7, Canada, ${ }^{3}$ Department of Anatomy \& Cell Biology, Schulich School of Medicine \& Dentistry, Western \\ University, London, Ontario N6A 3K7, Canada, ${ }^{4}$ Departments of Neurology, Neuroscience, Biomedical Engineering, and Center for Visual Science, \\ University of Rochester, Rochester, New York 14642, ${ }^{5}$ Departments of Physiology and Pharmacology, Psychology, Western University, London, \\ Ontario N6A3K7, Canada, ${ }^{6}$ Robarts Research Institute, Western University, London, Ontario N6A3K7, Canada, and ${ }^{7}$ Departments of Statistical and \\ Actuarial Sciences, and Computer Science, Western University, London, Ontario N6A3K7, Canada
}

How is the primary motor cortex (M1) organized to control fine finger movements? We investigated the population activity in M1 for single finger flexion and extension, using $7 \mathrm{~T}$ functional magnetic resonance imaging (fMRI) in female and male human participants and compared these results to the neural spiking patterns recorded in two male monkeys performing the identical task. fMRI activity patterns were distinct for movements of different fingers, but were quite similar for flexion and extension of the same finger. In contrast, spiking patterns in monkeys were quite distinct for both fingers and directions, which is similar to what was found for muscular activity patterns. The discrepancy between fMRI and electrophysiological measurements can be explained by two (non-mutually exclusive) characteristics of the organization of finger flexion and extension movements. Given that fMRI reflects predominantly input and recurrent activity, the results can be explained by an architecture in which neural populations that control flexion or extension of the same finger produce distinct outputs, but interact tightly with each other and receive similar inputs. Additionally, neurons tuned to different movement directions for the same finger (or combination of fingers) may cluster closely together, while neurons that control different finger combinations may be more spatially separated. When measuring this organization with fMRI at a coarse spatial scale, the activity patterns for flexion and extension of the same finger would appear very similar. Overall, we suggest that the discrepancy between fMRI and electrophysiological measurements provides new insights into the general organization of fine finger movements in M1.

Key words: electrophysiology; fingers; fMRI; hand control; humans; monkeys; primary motor cortex

\section{Significance Statement}

The primary motor cortex (M1) is important for producing individuated finger movements. Recent evidence shows that movements that commonly co-occur are associated with more similar activity patterns in M1. Flexion and extension of the same finger, which never co-occur, should therefore be associated with distinct representations. However, using carefully controlled experiments and multivariate analyses, we demonstrate that human fMRI activity patterns for flexion or extension of the same finger are highly similar. In contrast, spiking patterns measured in monkey M1 are clearly distinct. This suggests that populations controlling opposite movements of the same finger, while producing distinct outputs, may cluster together and share inputs and local processing. These results provide testable hypotheses about the organization of hand control in M1. 


\section{Introduction}

Dexterous movements of fingers require accurate coordination of different hand muscles. Hand muscles are innervated by motor neurons in the ventral horn of the spinal cord, which receive direct and indirect projections from the hand region of the contralateral primary motor cortex (M1; Lemon, 2008). In monkey species capable of better finger individuation, direct (monosynaptic) projections from M1 to ventral horn motor neurons are more pronounced (Heffner and Masterton, 1983; Bortoff and Strick, 1993). Lesions to the corticospinal tract (Tower, 1940; Lawrence and Kuypers, 1968; Lawrence and Hopkins, 1976; Sasaki et al., 2004) or to M1 (permanent: Liu and Rouiller, 1999; Darling et al., 2009; reversible: Schieber and Poliakov, 1998) result in a significant loss of finger individuation. Such symptoms are also reported in human stroke patients who have damage to the hand area of $\mathrm{M} 1$ or the descending corticospinal pathway (Lang and Schieber, 2003; Xu et al., 2017). These results indicate that M1 is important for the fine control of individuated finger movements.

What is less well understood is how this cortical control module for finger movements is organized. Here, we studied this question by investigating cortical activation patterns evoked during flexion and extension of individual fingers. Previous electrophysiological work in macaque monkeys (Schieber and Hibbard, 1993; Schieber and Poliakov, 1998) has indicated that motor cortical neurons have complex tuning functions, often responding to movements of multiple fingers and to both flexion and extension movements. Therefore, there exists no clearly organized "map," with separate regions dedicated to the control of a single finger. Instead, the population of M1 neurons involved in hand control must be organized by some other principle.

One plausible principle is that the statistics of natural hand use shapes the organization of neuronal populations in the hand region of M1. This idea predicts that movements that commonly co-occur in everyday life are represented in overlapping substrates in M1 (Graziano and Aflalo, 2007). In humans, fingers with high correlations between their joint-angle velocities during everyday hand movements (Ingram et al., 2008) have been shown to have more similar M1 activity patterns, as measured with functional magnetic resonance imaging (fMRI; Ejaz et al., 2015). The correlation structure of everyday finger movements nearly fully explained the relative similarities of M1 finger activity patterns and fit the data better than a model that used the similarity of the required muscle activity patterns (i.e., predicting that movements that use similar muscles also have similar activity patterns) or a somatotopic model (i.e., predicting that fingers are represented in an orderly finger map).

In this paper, we asked to what degree this kinematic hypothesis could generalize to movements of the same finger in different directions. We measured the activity evoked in the hand area of M1 using high-field fMRI while human participants performed near-isometric single finger flexion and extension presses with their right hand. By extrapolating the model used in the study by Ejaz et al. (2015) to this situation, we predicted that each movement should have its own, clearly separated representation in $\mathrm{M} 1$, as flexion and extension movements of the same finger can never co-occur. Indeed, it has been recently suggested that human motor cortex has multiple representations of each finger, one dedicated to flexion and one to extension (Huber et al., 2020).

We found, however, that the measured M1 fMRI patterns for flexion and extension of the same finger were strikingly similar, much more similar than would be expected for two movements that cannot co-occur. This similarity was not the result of cocontraction during the task. To better understand these results, we investigated the representational structure of single-neuron activity in M1 of two macaque monkeys trained on the same flexion-extension task (Schieber and Rivlis, 2005, 2007). The spiking patterns in monkeys were quite distinct for fingers and directions. From these results, we propose two, non-mutually exclusive hypotheses about the organization of finger movement representations in the primary motor cortex.

\section{Materials and Methods}

Human participants

Nine healthy participants were recruited for the study (five males and four females; mean age, 24.78 years; SD, 4.68; mean Edinburgh handedness score, 90.11; SD, 11.34). Participants completed three experimental sessions. During the first training session, participants learned to perform the finger individuation task. In the scanner session, participants performed the finger individuation task while undergoing fMRI. In the electromyography (EMG) session, participants performed the finger individuation task while muscle activities were recorded. All participants provided informed consent before the beginning of the study, and all procedures were approved by the Office for Research and Ethics at Western University.

\section{Experimental design of human finger individuation task}

In all three sessions (training, scanning, and EMG), the five fingers of the right hand were individually clamped between two keys (Fig. 1A). Foam padding on each key ensured that each finger was comfortably restrained. Force transducers (dynamic range, $0-16 \mathrm{~N}$; resolution, $<0.02$ $\mathrm{N}$, sampling rate, $200 \mathrm{~Hz}$; FS series, Honeywell) above and below each key monitored the forces applied by each finger in the extension and flexion directions.

During the task, participants viewed a screen that presented two rows of five bars (Fig. 1B). These bars corresponded to flexion or extension direction for each of the five fingers of the right hand. The forces applied by each finger were indicated on the visual display as five solid white lines (one per finger). On each trial, participants were cued to make an isometric, single finger flexion or extension press at one of three forces levels $(1,1.5$, or $2 \mathrm{~N}$ for extension; $1.5,2$, or $2.5 \mathrm{~N}$ for flexion) through the display of a white target box (Fig. 1B). Extension forces were chosen to be lower than flexion forces, as extension finger presses are more difficult (Valero-Cuevas et al., 1998; Li et al., 2003) and can lead to more enslaving (i.e., coarticulation) of noninstructed fingers (Yu et al., 2010). This design yielded two levels of matched target forces for flexion and extension presses ( 1.5 and $2 \mathrm{~N}$ ). The forces were similar to the low forces required in the monkey task design. The finger displacement required to achieve these force thresholds was minimal, such that the finger presses were close to isometric.

Each trial lasted $6000 \mathrm{~ms}$ and consisted of the following four phases (Fig. $1 B)$ : a cue phase $(1500 \mathrm{~ms})$, a press phase $(2000 \mathrm{~ms})$, a hold phase $(1000 \mathrm{~ms})$, and a $1500 \mathrm{~ms}$ intertrial interval. This trial structure was designed to mirror the nonhuman primate (NHP) task (see NHP methods). During the cue phase, a white box appeared in 1 of the 10 finger bars presented on screen, indicating the desired finger and direction. The desired pressing force was reflected by the relative location of the cue within the finger bar. After $1500 \mathrm{~ms}$, the cue turned green. This instructed the participant to initiate the finger press. Participants had up to $2000 \mathrm{~ms}$ after the cue turned green to reach the specified force. Once the pressing force was within the target box (target force, $\pm 12.5 \%$ ), the cue turned blue. Participants were trained to hold the force constant within this interval for $1000 \mathrm{~ms}$. When this time had elapsed, the cue disappeared and the participants were instructed to release the press by relaxing their hand. Importantly, participants were instructed not to actively move the finger in the opposite direction. A new trial started every $6 \mathrm{~s}$. For the scanning session, periods of rest were randomly intermixed between trials (see below). The muscle recording sessions lacked these rest periods, but otherwise had the same trial structure. 
Trials of the 30 conditions (five fingers $\times$ two directions $\times$ three forces) were presented in a pseudorandom order. Trials were marked as errors if the participant was too slow (i.e., did not initiate movement within $2000 \mathrm{~ms}$ of the go-cue), pressed the wrong finger or in the wrong direction, or if the participant did not reach at least $0.5 \mathrm{~N}$ force with the cued finger in the cued direction. Because of the pretraining, the participants had low error rates in both the fMRI (mean \pm SEM error rate across conditions, $1.48 \pm 1.05 \%)$ and EMG (mean \pm SEM error rate across conditions, $1.30 \% \pm 0.97 \%$ ) sessions, and accurately produced the required target forces (fMRI: mean peak force accuracy, $108.93 \pm 2.56 \%$ of the target forces; EMG: mean accuracy, $107.80 \pm 2.19 \%$ ). Therefore, we included all trials in subsequent analyses.

We also did not exclude any trials based on finger coactivation. Overall, participants were able to individuate their fingers relatively well. During fMRI extension trials, the forces applied through the noninstructed fingers were, on average, $14.01 \pm$ $1.41 \%$ of the forces applied by the instructed finger. During fMRI flexion, forces produced by noninstructed fingers were $20.51 \pm 1.49 \%$ of the force produced by the instructed finger. Most enslaving occurred during presses of the middle, fourth, and little fingers, all of which are difficult to individuate (Schieber, 1991). Note, however, that the presence of enslaving does not compromise the main finding of our study. To some degree, neural activity patterns related to flexion and extension of single fingers will always depend on the biomechanical coupling between fingers, either because the cortical activation patterns need to overcome that coupling or because coupling does occur, which then influences the recurrent sensory input. Our main conclusions are based on comparisons between flexion and extension presses, and remain valid whether we study the actions of isolated fingers, or groups of fingers (see Discussion).

\section{fMRI acquisition and analysis}

Image acquisition. We used high-field fMRI [7T Magnetom Scanner with a 32-channel head coil, Siemens (used at Western University)] to measure the blood oxygenation level-dependent (BOLD) responses in human participants. For each participant, evoked BOLD responses were measured for isometric single finger presses in the flexion and extension directions.

There were two repeats of each condition during each imaging run (five fingers $\times$ two directions $\times$ three force levels $\times$ two repeats $=60$ trials). The trial order in each run was randomized. In addition, five rest conditions of $6000 \mathrm{~ms}$ were randomly interspersed between trials within each run. Each run lasted $\sim 390$ s. Participants performed eight such runs during the scanning session.

During each run, 270 functional images were obtained using a multiband 2D echoplanar imaging sequence [GRAPPA (generalized autocalibrating partial parallel acquisition); in-plane acceleration factor, 2; multiband factor 2; repetition time (TR), $1500 \mathrm{~ms}$, echo time, $20 \mathrm{~ms}$; flip angle, $45^{\circ}$ ]. Per image, we acquired 32 interleaved slices (without gap) with an isotropic voxel size of $1.5 \mathrm{~mm}$. The first two images in the sequence were discarded to allow magnetization to reach equilibrium. To estimate magnetic field inhomogeneities, we acquired a gradient echo

\section{B Experimental paradigm (human)}

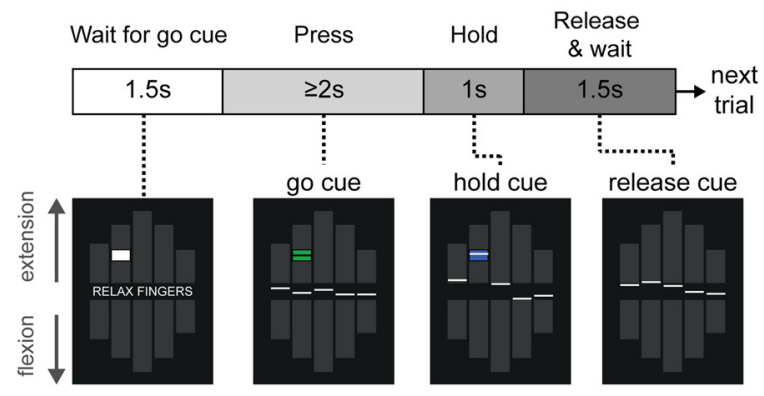

D Experimental paradigm (monkey)

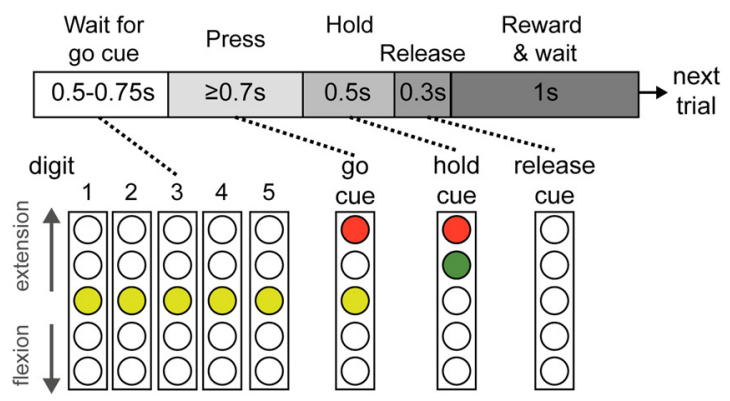

Figure 1. Experiment paradigms. $\boldsymbol{A}$, Human participants made isometric single finger presses in the flexion and extension sere what finger and what direction to press. The monkeys had up to $700 \mathrm{~ms}$ from the onset of the go-cue to press the cued finger in the cued direction. They were trained to hold the press for $500 \mathrm{~ms}$ before relaxing the finger.

field map at the end of the scanning session. Finally, a T1-weighted anatomic scan was obtained using an MPRAGE (magnetization-prepared rapid gradient echo sequence) with a voxel size of $0.75 \mathrm{~mm}$ isotropic (3D gradient echo sequence; TR, $6000 \mathrm{~ms}$; 208 volumes).

Image preprocessing and first-level analysis. Functional images were first realigned to correct for head motion during the scanning session (three translations: $\mathrm{x}, \mathrm{y}, \mathrm{z}$; three rotations: pitch, roll, yaw), and coregistered to each participant's anatomic T1-weighted image. Within this process, we used a B0 fieldmap to correct for image distortions arising from magnetic field inhomogeneities (Hutton et al., 2002). Because of the relatively short TR (1.5 s), no slice-timing correction was applied. Nor were the data spatially smoothed or normalized to a standard template.

The minimally preprocessed data were then analyzed using a general linear model (GLM; Friston et al., 1994) using SPM12 (https://www.fil. ion.ucl.ac.uk/spm/). Each of the finger-direction-force conditions was modeled with separate regressors per run, resulting in 30 regressors per run $(30 \times 8$ runs $=320$ task regressors $)$, along with an intercept for each run. The regressor was a boxcar function that started at the presentation of the go-cue and lasted for the trial duration, spanning the press, hold, and release periods of each trial. The boxcar functions were convolved with a hemodynamic response function with a delayed onset of $1000 \mathrm{~ms}$ and a poststimulus undershoot at $7500 \mathrm{~ms}$. Given the low error rate, we did not exclude any trials from this analysis. To model the long-range temporal autocorrelations in the functional timeseries, we used the SPM FAST autocorrelation model with restricted maximum likelihood estimation (for details, see Arbuckle et al., 2019). High-pass filtering was 


\section{Left M1 fMRI activity patterns (2N presses)}
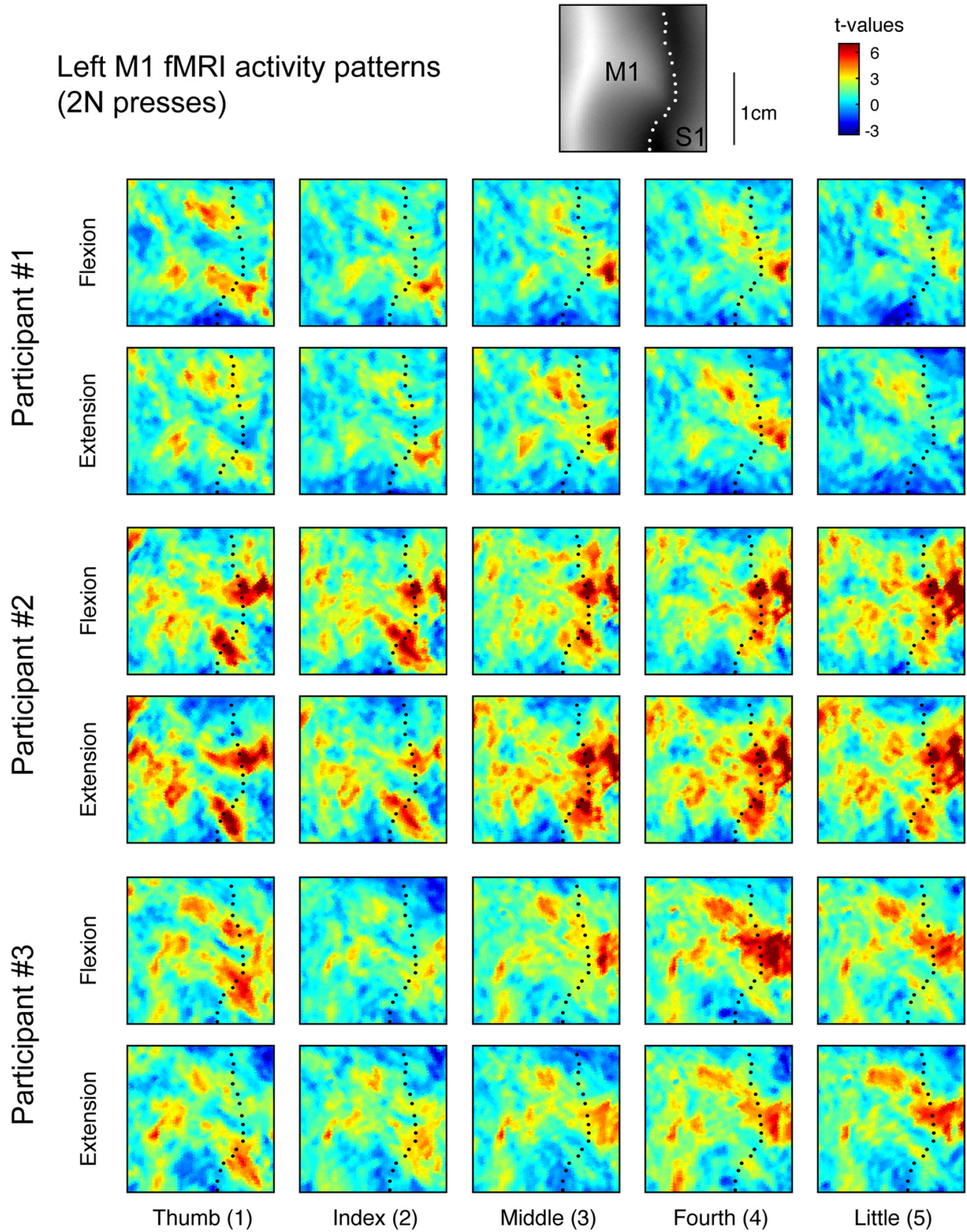

Middle (3)

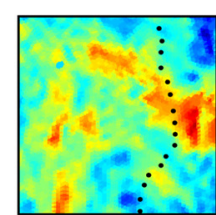

Fourth (4)

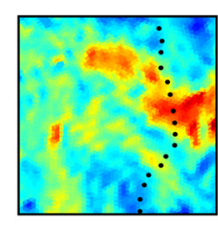

Little (5)

Figure 2. fMRl activity patterns for finger flexion and extension in human M1. Evoked fMRI activity maps ( $t$ values) for three participants for each of the five fingers pressing in the extension and flexion directions at 2 N. Results were normalized to a surface-based atlas. Maps are shown in the hand-knob region of the left (contralateral) hemisphere. The black dotted line shows the fundus of the central sulcus. The top inset shows the average sulcal depth.

then achieved by temporally prewhitening the functional data with this temporal autocorrelation estimate. This analysis resulted in one activation estimate (" $\beta$-weights") for each of the 30 conditions per run for each participant. For visual display (Fig. 2) and further analysis, the $\beta$ values were divided by the root mean square error from the first-level GLM to yield a $t$ value per voxel for each condition in each run.

Surface reconstruction and ROI definition. Each participant's T1weighted image was used to reconstruct the pial and white matter/gray matter surfaces using Freesurfer (Fischl et al., 1999a). Individual surfaces were aligned across participants and spherically registered to match a template atlas (Fischl et al., 1999b) using a sulcal-depth map and local curvature as minimization criteria. M1 was defined as a single region of interest (ROI) on the group surface using probabilistic cytoarchitectonic maps aligned to the template surface (Fischl et al., 2008). We defined M1 as being surface nodes with the highest probability for Brodmann area 4 that fell within $1.5 \mathrm{~cm}$ above and below the hand knob anatomic landmark (Yousry et al., 1997). To avoid cross-contamination between M1 and S1 activities along the central sulcus, voxels with $>25 \%$ of their volume in the gray matter on the opposite side of the central sulcus were excluded.

Multivariate fMRI analysis. We used the cross-validated squared Mahalanobis dissimilarity (i.e., crossnobis dissimilarity) to quantify differences between fMRI activity patterns for each pressing condition within each participant (Walther et al., 2016; Diedrichsen et al., 2020). Cross-validation ensures the dissimilarity estimates are unbiased, such that if two patterns differ only by measurement noise, the mean of the estimated dissimilarities would be zero. This also means that estimates can sometimes become negative (Diedrichsen et al., 2016). Therefore, dissimilarities significantly larger than zero indicate that two patterns are reliably distinct.

The fMRI activity patterns were first-level GLM $\beta$-weights for voxels within the M1 ROI mask. Analyses were conducted using functions 
from the representational similarity analysis (RSA; Nili et al., 2014) and PCM (Diedrichsen et al., 2018) MATLAB toolboxes. The crossnobis dissimilarity $d$ between the fMRI activity patterns $(x)$ for conditions $i$ and $j$ was calculated as follows:

$$
d_{i, j}=\frac{1}{M} \sum_{m}^{M}\left(x_{i}-x_{j}\right)_{m}^{T} \Sigma^{-1}\left(x_{i}-x_{j}\right)_{\sim m}
$$

where the activity patterns from run $m$ are multiplied with the activity patterns averaged over all runs except $m(\sim m)$. $\Sigma$ is the voxelwise noise covariance matrix, estimated from the residuals of the GLM, and slightly regularized to ensure invertibility. Multivariate noise normalization removes spatially correlated noise and yields generally more reliable dissimilarity estimates (Walther et al., 2016).

The dissimilarities are organized in a representational dissimilarity matrix (RDM). The RDM is a symmetric matrix (number of conditions $\times$ number of conditions in size) with off-diagonal values corresponding to the paired distance between two conditions. Values along the diagonal are zero, as there is no difference between a pattern paired with itself.

We calculated an RDM for the matched force conditions separately (i.e., the 1.5 and $2 \mathrm{~N}$ presses, 10 conditions each), and then averaged the resulting RDMs within each participant. This yielded one RDM per participant containing the crossnobis dissimilarities between presses of the five fingers in either direction ( 10 conditions, 45 dissimilarity pairs).

Estimating spatial tuning of fingers and direction. We considered the possibility that fingers and directions could be encoded at different spatial scales in M1. We therefore estimated the spatial covariance of tuning for fingers and directions. Within each imaging run, we averaged the fMRI activity patterns ( $t$ values) for each condition across the matched forces ( 1.5 and $2 \mathrm{~N}$ ). This yielded a vector of 10 activity values per voxel (one value per each finger per direction), which we refer to as an "activity profile." We modeled the activity profile values $\left(y_{i, j}\right)$ of each voxel and partition using three components, as follows:

$$
y_{i, j}=f_{i}+d_{j}+q_{i, j}
$$

where $f_{i}$ is the main effect of finger $i, d_{j}$ is the main effect of direction $j$, and $q_{i, j}$ is the finger $\times$ direction interaction effect. We used ordinary least-squares regression to estimate the finger and direction components. The residual from the regression was taken as an estimate of the interaction component.

We first reconstructed the activity profiles using only the finger component $(f)$, and then estimated the covariance of the finger activity profiles between voxel pairs in M1. These covariances were calculated in a cross-validated fashion, as follows: we averaged the reconstructed activity profiles for odd and even runs separately, and then then computed the covariance of the activity profile of different voxels across independent partitions of the data. Given that the estimates for all components contained some noise, normal covariance estimates are biased by the spatial structure of the noise. Cross-validation alleviates the influence of noise on covariance/variance estimation, as the average of the product of noise across odd and even runs is zero.

We then binned the covariances based on the spatial distance between each voxel pair and averaged the covariances within each bin. The first bin included only the cross-partition covariance between each voxel and itself (i.e., the cross-validated estimate of the voxel variances). The second bin contained the covariances between immediately and diagonally neighboring voxels $(1.5-2.6 \mathrm{~mm})$, the third bin the second layer of direct and diagonally neighboring voxels $(>2.6-5.2 \mathrm{~mm})$, and so on, up to a total distance of $20.8 \mathrm{~mm}$. Finally, we normalized the binned covariances by the cross-validated voxel variances (value of the first bin) to obtain an estimate of the spatial autocorrelation function (ACF) for fingers in M1.

We used the same procedure to estimate the ACF for direction. Importantly, we included both the direction $(d)$ and the finger $\times$ direction interaction $(q)$ components in the activity profile reconstruction. We included the interaction component as we hypothesized that the tuning of voxels to flexion and extension patterns would be different across fingers.
Finally, we estimated the smoothness of the finger and direction ACFs (Diedrichsen et al., 2011). To do this, we fitted a function that decayed exponentially with the square of the distance $(\delta)$ between voxels $(v)$, as follows:

$$
\operatorname{ACF}\left(v_{x}, v_{x+\delta}\right)=\exp \left(-\frac{\delta^{2}}{2 s^{2}}\right)
$$

Here, $s$ is the SD of the ACF. If neighboring voxels are relatively independent (i.e., low covariance), the value of $s$ will be small. While we can use $s$ to express the smoothness of the ACF, the smoothness can also be expressed as the full-width at half-maximum (FWHM) of the Gaussian smoothing kernel that-when applied to spatially independent datawould yield the same ACF. The SD of this Gaussian kernel is $\sqrt{1 / 2 s}$, and the FWHM is calculated as follows:

$$
F W H M=2 s \sqrt{\log (2)}
$$

We applied this approach to the reconstructed finger and direction activity profiles separately to estimate the FWHM of fingers and direction M1. The goodness of fit (evaluated with $R^{2}$ ) of the fitted exponential decays were both high (mean \pm SEM $R^{2}$ of finger ACF, $0.960 \pm 0.008$; mean \pm SEM $R^{2}$ of direction ACF, $\left.0.908 \pm 0.020\right)$. Although there was a significant difference between the finger and direction model $R^{2}$ (twosided paired $t$ test: $\left.t_{(8)}=2.412, p=0.0424\right)$, the mean difference was quite small (mean \pm SEM, $0.052 \pm 0.021$ ).

Center of gravity analysis. We analyzed the activity patterns to determine whether there were significant differences in the spatial arrangement of finger flexion and extension, as proposed by Huber et al. (2020). To ensure that our analysis closely matched this previous report, we restricted the center of gravity $(\mathrm{CoG})$ analysis to include only surface nodes from Brodmann area $4 \mathrm{a}$, as based on the probabilistic atlas (Fischl et al., 2008). We also restricted the analysis to the hand region by selecting only vertices within $1.5 \mathrm{~cm}$ of the hand knob anatomic landmark. On the flattened activity maps for each finger, we then calculated the CoG of each map as the average spatial location $(\hat{x}, \hat{y})$ of each surface node $(i)$, weighted by its respective $t$ value $(t)$, as follows:

$$
\begin{gathered}
\hat{x}=\frac{\sum_{i=1}^{P} x_{i} t_{i}}{\sum_{i=1}^{P} t_{i}} \\
\hat{y}=\frac{\sum_{i=1}^{P} y_{i} t_{i}}{\sum_{i=1}^{P} t_{i}},
\end{gathered}
$$

In the above calculations, we set negative $t$ values equal to zero, thereby focusing our spatial analysis on regions that showed activity increases. We used a two-factor repeated-measures multivariate ANOVA (MANOVA) to test for significant differences between the measured CoGs for different fingers and directions. To summarize the structure of the spatial arrangement, we calculated the pairwise Euclidean distances between the CoG coordinates for each condition and arranged them into an RDM.

\section{EMG recording and analysis}

EMG recordings and preprocessing. In a separate session, we recorded hand and forearm muscle activity to ensure that participants performed the task as instructed. During the EMG session, participants were seated upright, whereas during the fMRI session participants lay prone in the scanner. In both sessions, however, we ensured that the arm was in a relaxed position, the palm of the hand was supported by the device, the wrist was slightly extended, and the elbow joint was slightly bent. Thus, wrist and forearm posture, both known to influence muscle activity during finger movements (Mogk and Keir, 2003; Beringer et al., 2020), were matched across the two sessions. Participants' skin was cleaned with rubbing alcohol. Surface EMG of distal muscles of the hand 
were recorded with self-adhering $\mathrm{Ag} / \mathrm{AgCl}$ cloth electrodes (Kendall H59P-127 repositionable monitoring electrodes, CardinalHealth). Electrodes were cut and positioned in line with a muscle in a bipolar configuration with an approximate $1 \mathrm{~cm}$ interelectrode distance. The surface EMG of proximal limb muscles was recorded with surface electrodes (Bagnoli-8 system with DE-2.1 sensors, DELSYS). The contacts were coated with a conductive gel. Ground electrodes were placed on the ulna at the wrist and elbow. The signal from each electrode was sampled at $2000 \mathrm{~Hz}$, de-meaned, rectified, and low-pass filtered (fourth-order Butterworth filter, $f_{c}=40 \mathrm{~Hz}$ ).

Multivariate EMG analysis. We used the crossnobis dissimilarity to quantify differences between patterns of muscle activities for each movement condition, similar to the fMRI analysis. This metric is invariant to the scaling of the EMG signals from each electrode and has been established in previous work (Ejaz et al., 2015). Briefly, we first calculated the average square root EMG activity for each electrode and trial by averaging over the press and hold time windows [mean window, $1800 \mathrm{~ms}$ (up to a maximum window of $3000 \mathrm{~ms}$ )]. We then subtracted the mean value for each electrode across conditions for each run independently to remove any drifts in the signal. These values were then divided by the SD of that electrode across trials and conditions to avoid arbitrary scaling. Finally, we calculated the crossnobis dissimilarity between pairs of EMG activity patterns for different conditions across runs.

\section{Experimental design of monkey finger individuation task}

The behavioral task performed by two male Macaca mulatta monkeys (monkeys C and G) has been described previously (Schieber, 1991; Schieber and Rivlis, 2007). Briefly, the monkeys were trained to perform cued single finger flexion and extension presses. Each monkey sat in a primate chair and, similar to the human device described above, their right hand was clamped in a device that separated each finger into a different slot (Fig. 1C). Each slot was composed of two microswitches (one in the flexion direction and one in the extension direction). One switch was closed by flexing the finger, the other by extending the finger. The absolute degree of movement required to close either switch was minimal (a few millimeters), and therefore the force required to make and hold a successful press was small-similar to the human finger individuation task. Therefore, like the fMRI task behavior, these finger movements are very close to isometric presses.

A series of LED instructions were presented to the monkey during each trial (Fig. 1D). A successful trial occurred when the monkey pressed the cued finger in the cued direction without closing any other switch. Similar to our human experiment design, the monkeys were trained to hold the cued switch closed for $500 \mathrm{~ms}$ before relaxing the finger (Fig. $1 D$ ). At the end of a successful trial, the monkey received a water reward. The wrist of the monkey was also clamped in this device, and some trials required the monkey to flex or extend the wrist. Wrist trials were not included in the current analysis. Flexion and extension trials of each finger and wrist were pseudorandomly ordered. In the case of a behavioral error, trials were repeated until successful. Therefore, we excluded all trials with an error and also the successful trials that followed error trials to avoid potential changes in the baseline firing rate of the recorded neuron.

In contrast to the human task, the required force level for the monkeys was the same for all trials; therefore, they did not receive continuous visual feedback about the force produced. Instead, they received small tactile feedback when the switch closed, a feature that was absent from the human task. Despite these small differences in feedback, the task requirements were well matched across species: both monkeys and humans were required to produce low, well controlled forces with a single finger, while keeping forces on the noninstructed fingers minimal, to avoid either unwanted switch closure or excessive movement of the associated visual feedback.

\section{Analysis of single-cell spiking data}

Spike rate calculation. Single cells were isolated and spike times were recorded while monkeys performed the finger individuation task. The details of the recordings have been reported previously (Poliakov and Schieber, 1999). Each trial was labeled with a series of behavioral markers, indicating the time of trial onset, presentation of condition cue, switch closure, and reward onset. For the spike rate traces (see Fig. 4), we calculated the spike rate per $10 \mathrm{~ms}$ bin, aligned to press onset, and smoothed the binned rates with a Gaussian kernel (FWHM $=50 \mathrm{~ms}$ ). For the dissimilarity analysis (see below), we calculated the average spike rate over time per trial starting at go-cue onset (when the monkey was instructed as to which finger and direction to press) until the end of the hold phase $(500 \mathrm{~ms}$ after switch closure). This time window encompassed a short period of time before the start of the finger press and the entire hold duration of the press (monkey C: mean window, $739 \mathrm{~ms}$; monkey G: mean window, $773 \mathrm{~ms}$ ).

Multivariate spiking analysis. Similar to the human fMRI and EMG analyses, we computed crossnobis dissimilarities between spiking patterns for different conditions within each monkey. To crossvalidate the estimated distances, we restricted our analysis to include cells for which we had at least two successful trials for each finger in both directions. This criteria yielded 44,801 trials from 238 cells in monkey C (median number of trials per cell, 168; median number of trials per condition per cell, 19) and 5535 trials from 45 cells in monkey G (median number of trials per cell, 115; median number of trials per condition per cell, 12). After calculating the average spike rates, we arranged the spike rates into vectors per condition (see Fig. 4B). To account for the Poisson-like increase of variability with increasing mean firing rates, we applied the square root transform to the average firing rates ( $\mathrm{Yu}$ et al., 2009).

For each cell per condition, we randomly split the square root spike rates from different trials into one of two partitions. The random splits contained approximately the same number of trials, which ensured that each condition was approximately equally represented in each partition. We then averaged the spike rates within each partition. This yielded two independent sets of spiking patterns per monkey (10 patterns: 5 fingers $\times$ 2 directions). Per partition, we normalized spike pattern of each neuron by dividing by the maximum rate of the neuron across conditions, and then reweighted the normalized spike rates per cell according to the number of trials per cell (cells with more trials were upweighted; vice versa for cells with fewer trials). Finally, we calculated pairwise cross-validated Euclidean distances between the two sets of patterns. We repeated this RDM calculation procedure $1000 \times$ per monkey, each time using a different random partitioning of the data. We then averaged the RDMs across iterations to yield one RDM estimate per monkey. We note that results were not dependent on the normalization we chose-results were qualitatively consistent when using raw firing rates, $z$-scoring the firing rates, not applying trial reweighting, and various combinations of these approaches.

\section{Kinematic finger model RDM}

As in the study by Ejaz et al. (2015), we used the statistics of naturalistic hand movements to predict the relative similarity of single finger representations in M1. In the text, we refer to this model as the kinematic model. To construct the kinematic model RDM, we used hand movement statistics from an independent study in which six male participants wore a cloth glove imbedded with motion sensory (CyberGlove, Virtual Technologies) while they performed everyday activities (Ingram et al., 2008). These statistics included the velocities about joint angles specific to each of the five fingers of the participants' right hands. Positive velocities indicated finger flexion, and negative velocities indicated finger extension.

Because the movement in our finger pressing task was restricted to movements about the metacarpal (MCP) joint of each finger, we used the MCP joint velocities to predict cortical M1 finger similarity. First, we split the data for each joint velocity into the following two vectors: one for flexion and one for extension, taking the absolute of the velocities in this process. During periods of finger flexion, we set the extension velocity to zero, and vice versa. This resulted in 10 velocity vectors ( 5 fingers $\times 2$ directions). Then, to account for differences in scaling, we normalized each velocity vector to a length of 1 . Finally, we calculated the dissimilarities between pairs of these processed velocity vectors. We 
averaged these RDMs across the six participants in the natural statistics dataset, yielding one kinematic model RDM.

\section{Experimental design and statistical analysis}

Statistical analysis of dissimilarities. We summarized the RDMs by classifying dissimilarities into finger-specific and direction-specific dissimilarities for each participant and dataset. Finger-specific dissimilarities were the dissimilarities between conditions where different fingers were pressed in the same direction (10 pairs for flexion, 10 pairs for extension). Direction-specific dissimilarities were the dissimilarities between conditions where the same finger was pressed in different directions (five pairs total). Within each category, dissimilarities were averaged. For the human data, we used one-sided, one-sample $t$ tests to test whether mean finger and direction dissimilarities were greater than zero. To compare between the average finger and direction dissimilarities, we used two-sided paired $t$ tests. We report the mean and SE of the dissimilarities where appropriate in the text.

Statistical analysis of RDM correlations. Pearson's correlations between the vectorized upper triangular elements of the RDMs were used to compare different RDMs (Ejaz et al., 2015). To calculate the stability of RDMs, we calculated the Pearson's correlations between all possible pairs of the participants' RDMs. This yielded 36 correlations ( 1 per unique participant pair). We Fisher $z$-transformed these correlations and calculated the mean and SE. We used these values to calculate the lower and upper bounds of the 95\% confidence interval, assuming normality. Finally, the mean and confidence bounds were transformed back to correlations. We report these values in the text as $r=$ mean [lower bound - upper bound]. The same method was applied to correlations between measured RDMs and model predictions. Note that, because we used a within-subject design, the muscle model predictions were specific to each human participant. In contrast, the kinematic model prediction was the same for each participant because data for this model was obtained from an independent study. Paired $t$ tests were performed on Fisher $z$-transformed correlations to compare fits between models.

Estimating noise ceiling for RDM model fits. Since the dissimilarities between fMRI patterns can only be estimated with noise, even a perfect model fit would not result in a perfect correlation with the RDM of each participant. Therefore, we estimated the noise ceiling, which places bounds on the expected model correlations if the model is a perfect fit. We first calculated the average correlation of each participant's RDM with the group mean RDM (Nili et al., 2014), treating the mean RDM as the perfect model. The resulting average correlation is an overestimate of the best possible fit, as each RDM is correlated with a mean that includes that RDM (and hence also the measurement error of that RDM). To then estimate a lower bound, we calculated the correlation between a participant's RDM and the group mean RDM in which that individual was removed.

\section{Results}

\section{M1 fMRI activity patterns differ strongly for different} fingers, not for direction

We measured activity patterns evoked in M1 in human participants $(n=9)$ while they performed a near-isometric finger flexion-extension task in a 7T MRI scanner. Participants' right hands were clamped in a device that had force transducers mounted both above (extension) and below (flexion) each finger (Fig. 1A) to record forces produced at the distal phalanges. The device limited the overall degree of movement to a few millimeters, thereby making the task near isometric. On each trial, participants were cued to press a single finger in one direction, while keeping the other fingers as relaxed as possible (Fig. 1B). They had to reach the required force level, hold it for $1 \mathrm{~s}$, and then simply relax their hand to let the force passively return to baseline. This aspect of the task instruction was critical to ensure that participants did not activate the antagonist muscles during release.
Figure 2 shows the activity patterns measured in left M1 (contralateral to movement) for three participants during righthanded finger presses at $2 \mathrm{~N}$. As previously observed (Ejaz et al., 2015), the activity patterns did not consist of focal regions of activity dedicated to each finger. Rather, the spatial patterns were complex and involved multiple overlapping regions within the M1 hand area. Furthermore, the intersubject variability in the spatial organization of these patterns was considerable.

One common observation across all participants, however, was that the activity patterns were different between different fingers (e.g., index flexion vs fourth flexion), but rather similar for flexion and extension of the same finger (e.g., index flexion vs index extension). We used RSA to quantify these observations by calculating a measure of dissimilarity (crossnobis dissimilarity; see Materials and Methods) between each pair of fMRI patterns. Large dissimilarity values indicate that the two patterns are quite distinct with little overlap. A value of zero indicates that the two patterns are identical and differ only by noise. We restricted the analysis to conditions with matched force levels across flexion and extension. The group-averaged RDM is shown in Figure 3A. Both within the finger flexion and extension conditions, there was a characteristic structure with the thumb activity pattern being the most distinct and neighboring fingers tending to have more similar activity patterns. Across directions, activity patterns evoked by pressing the same finger in different directions were the most similar. This representational structure was quite stable across participants (average interparticipant Pearson's $r=0.790$; 95\% CI, 0.754-0.820).

To obtain predictions for flexion and extension movements, we needed to adapt the natural usage model, proposed by Ejaz et al. (2015). This model used kinematic finger data, specifically the joint-angle velocities of the MCP joints, that was recorded while subjects participated in their normal everyday tasks (Ingram et al., 2008, data).

Fingers were predicted to have more similar representations if their movement velocities, across flexion and extension, were positively correlated. For the current experiment, we split the data into periods of finger flexion and finger extension (see Materials and Methods), resulting in 10 time series and calculated the correlation between them (after taking the absolute value).

The estimated kinematic RDM (Fig. 3B) showed similar structures within flexion and extension movements. The thumb was the most distinct compared with the other fingers, and for the remaining fingers there was a clear similarity structure with neighboring fingers more similar than non-neighboring. This structure very closely mirrored those found for fMRI activity patterns: flexion and extension fMRI RDMs correlated strongly with the corresponding kinematic models for flexion $(r=0.727$; 95\% CI, 0.635-0.800) and extension ( $r=0.797$; 95\% CI, 0.6840.873 ) RDMs (Fig. 3C, white). Compared with the noise ceiling [Fig. 3C, gray bar (which reflects the best possible model fit given measurement noise) see Materials and Methods], the kinematic model accounted for $79.9 \%$ and $84.9 \%$ of the variance in the flexion and extension fMRI RDMs, respectively.

In contrast, the kinematic model completely failed to predict the relationships between activity patterns for flexion and extension. Because flexion and extension of the same finger can never co-occur, the kinematic model predicts that the movements are associated with quite distinct cortical activity patterns. The measured fMRI patterns, however, were rather similar for these two actions. As a result, the full kinematic model was not a good fit to the full fMRI RDM ( $r=0.086 ; 95 \%$ CI, 0.038-0.133), much below the noise ceiling ( $r=0.875$; 95\% CI, $0.822-0.913)$. 
A

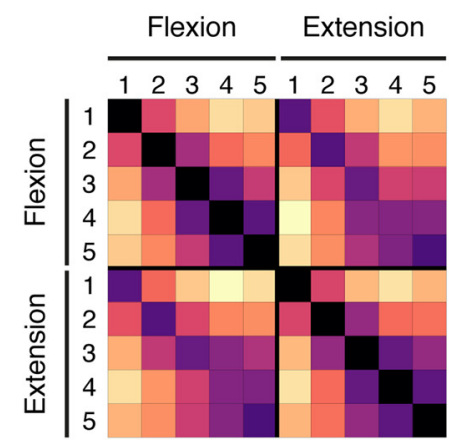

dissimilarity

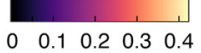

\section{Model fits}
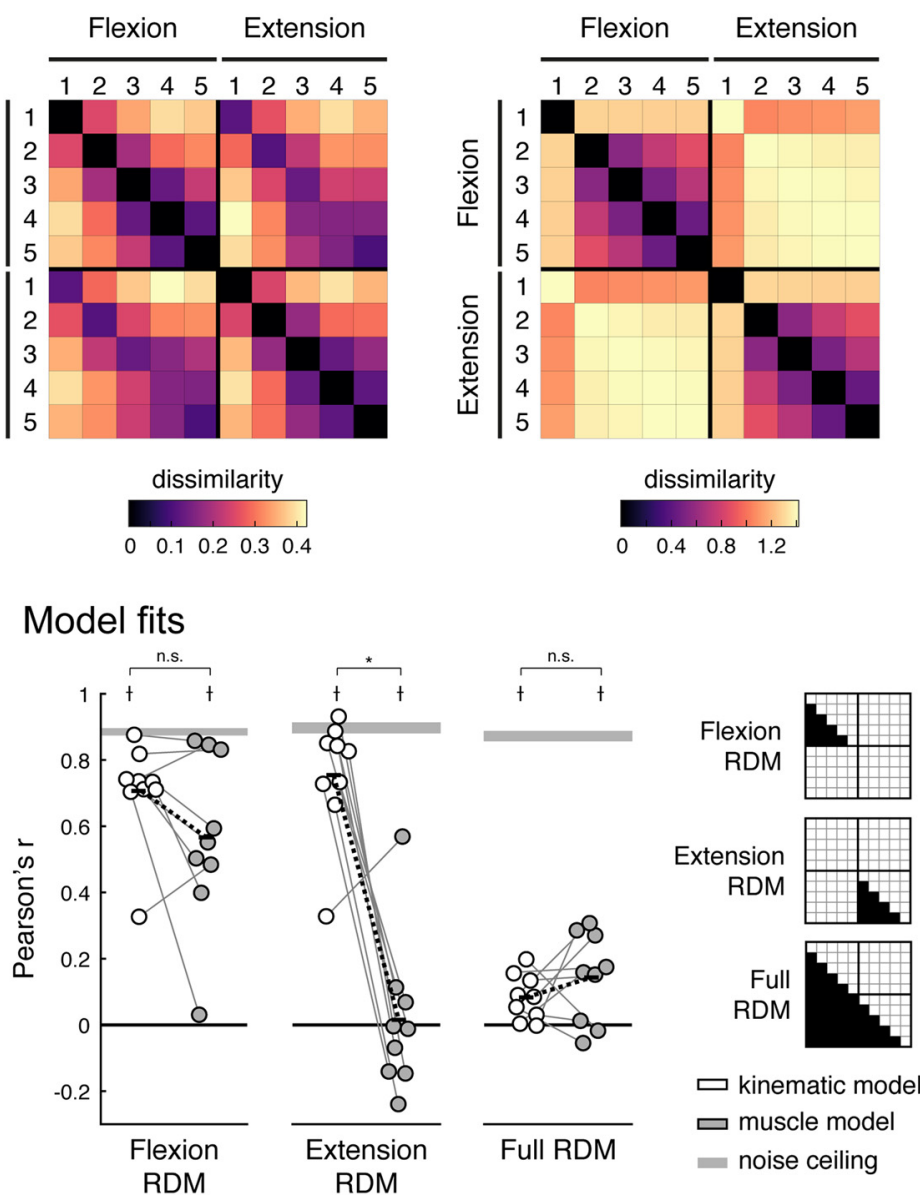

$\square$ kinematic model

$\square$ muscle model

noise ceiling

Figure 3. Representational structure of fingers and direction in human M1. $\boldsymbol{A}$, Group average of the fMRI RDM. $\boldsymbol{B}$, Predicted RDM from the kinematic model. To aid visual inspection, the values of the RDMs in $\boldsymbol{A}$ and $\boldsymbol{B}$ are plotted as the square root of the dissimilarities. All statistical analyses of the RDMs are done on squared distances. $C$, Model fits (Pearson's correlation) of the kinematic (white) and muscle (gray) models to the M1 RDM for flexion, extension, and the full RDMs (the indices for each RDM are shown on the right). The muscle model was specific to each participant and was estimated from the EMG data. The gray bars denote noise ceilings (theoretically, the best possible fits). Each dot reflects one participant, and thin gray lines connect fits of each model to the same participant. Black bars denote the means, and black dashed lines denoted the mean paired difference. $*$ Significant differences between model fits (one-sided paired $t$ test, $p<0.05$ ); ${ }^{\dagger}$ significantly lower than the noise ceiling (two-sided paired $t$ test, $p<0.05)$; n.s., not significant $(p>0.05)$.

Thus, although the statistics of movement co-occurrence was a good predictor for representational similarity between the activity patterns for different fingers (i.e., within flexion or extension), this simple model failed to predict the relative organization of the patterns for flexion and extension of the same finger. Although flexion and extension of the same finger cannot co-occur, their fMRI activity patterns were highly similar. In the remainder of this paper, we explore a number of possible explanations for this finding and propose a candidate model of the organization.

\section{Similarities of cortical representations for presses in different directions cannot be explained by the patterns of muscle activity}

We first considered the possibility that the structure of similarity between flexion and extension presses can be explained by the patterns of muscle activity required by these movements. Specifically, it is possible that participants cocontracted both agonist and antagonist muscles, or that they activated the antagonistic muscles when returning to baseline. Given the temporally sluggish nature of the BOLD signal measured with fMRI, either behavior could cause the cortical activity patterns evoked during flexion to resemble activity patterns during extension (and vice versa). Therefore, we conducted a control experiment with the same participants outside the MR scanner, during which we recorded surface EMG from 14 sites of the hand and forearm in the participants (Fig. 4A), while they performed the same isometric finger flexionextension task as in the fMRI session. Performance on the task was comparable to that during the fMRI scan.

As an example, the participant-averaged EMG data from an electrode placed above the abductor digiti minimi (ADM) muscle (Fig. $4 B$ ) showed that the ADM muscle was recruited only during the flexion of the little finger. During extension of the same finger, the muscle was silent, both during hold and release. In general, we found very little evidence for cocontraction of the antagonist muscle.

For a quantitative analysis, we averaged the muscle activity from the time of the go-cue to the end of the hold phase. The EMG patterns averaged across participants (Fig. 4C) already allow for two observations. First, the muscle activities for the same movement at different force levels were very similar and increased with increasing force. The average correlation across force levels for each fingerdirection combination was high, indicating that the same muscles were consistently recruited to perform the same finger press across different force levels (within participant correlations: $r=0.860$; 95\% CI, 0.808-0.898). Second, quite distinct muscle groups were recruited to produce forces with the same finger in different directions. The average correlation between the pattern of muscle activity recruited to press the same finger in different directions was low (within-participant correlations; $r=0.244$; 95\% CI, 0.150-0.334).

We then derived a muscle-based RDM by calculating the crossnobis dissimilarity between normalized activity patterns for each condition. As for the fMRI analysis, we included the patterns for the matched force conditions only. The group-averaged matrix RDM (Fig. $4 D$ ) was only moderately stable across participants (average interparticipant Pearson's $r=0.480$; 95\% CI, $0.379-0.570$ ), likely reflecting the fact that there was some degree of interindividual variation in electrode placement.

We tested to what degree the patterns of muscle activity, specific to each participant, could explain the cortical similarity structure between individual finger movements within the flexion or extension directions. For the flexion direction, the fit of the muscle model $(r=0.611 ; 95 \%$ CI, $0.408-0.757)$ was lower than that for the kinematic model in six of nine participants (Fig. $3 C$ ), but the difference did not reach statistical significance (one- 


\section{A EMG electrode sites}

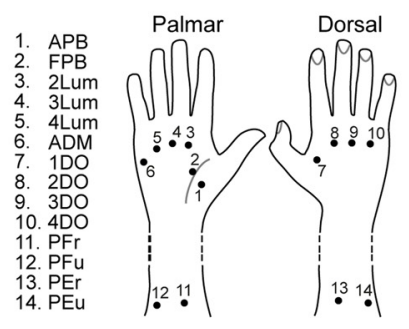

B Abductor digiti minimi EMG activity (group)

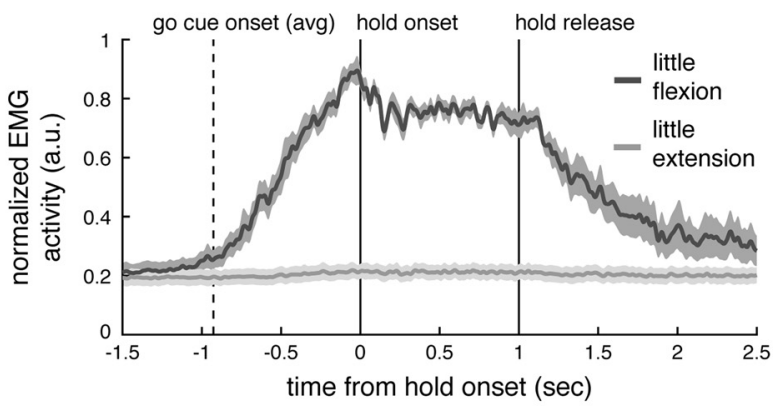

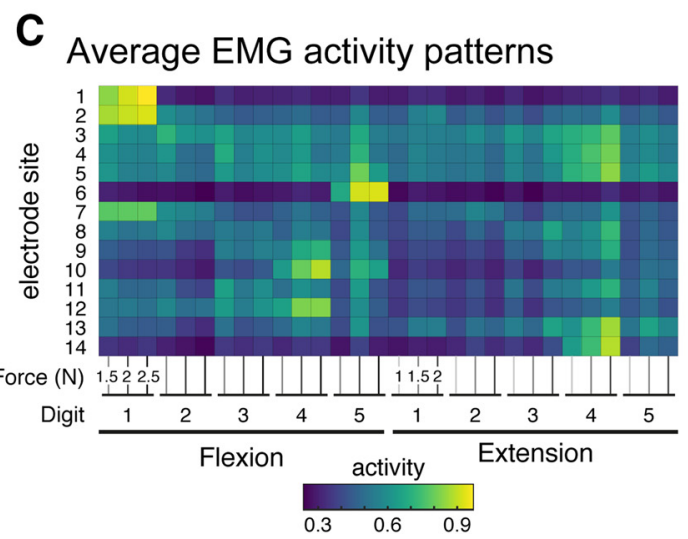

D Average muscle RDM

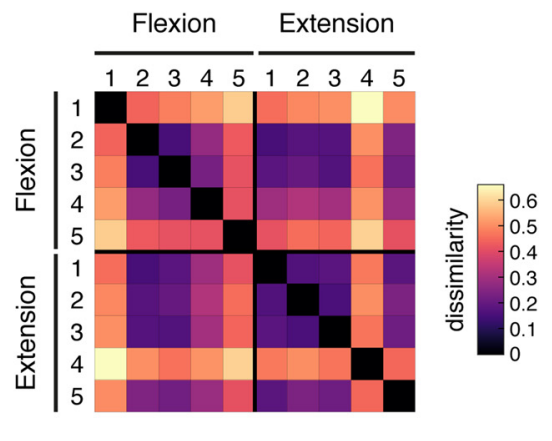

Figure 4. Quantifying similarity of muscle activity patterns during finger flexion and extension. $\boldsymbol{A}$, Fourteen surface electrode sites. $\boldsymbol{B}$, Group averaged normalized EMG (normalized, per participant, to peak activity from this electrode across trials) from the ADM muscle during $2 \mathrm{~N}$ little finger (5) flexion (dark gray) and extension (light gray) trials, aligned to hold onset (0 s). During extension movement (light gray trace, $>1000 \mathrm{~ms}$ ), this flexor muscle was not recruited. Shaded areas reflect SEM. Traces were smoothed with a Gaussian kernel (FWHM, 25ms). C, Average muscle activity across participants, normalized by peak activation across conditions (per participant), recorded from the 14 electrode sites during the flexion extension task. Each condition was measured under three force conditions. D, Group average RDM of the muscle activity patterns. As in Figure 2, the RDM is plotted as square root dissimilarities to aid visual inspection.

sided paired $t$ test kinematic $>$ muscle: $t_{(8)}=1.775, p=0.0569$ ). For the extension direction, the muscle model fit substantially worse ( $r=0.020 ; 95 \% \mathrm{CI},-0.147$ to 0.187 ), significantly less than the kinematic model (one-sided paired $t$ test kinematic $>$ muscle: $\left.t_{(8)}=5.588, p=2.59 \mathrm{e}-4\right)$. This generally confirms the results reported in the study by Ejaz et al. (2015) that the relative similarities of M1 finger flexion activity patterns are better explained by the correlation structure of everyday movements than the correlation structure of the required muscle activity patterns. Our new results now show that this observation generalized also to extension movements.

Critically, however, the muscle activity model did not provide a good explanation for the similarity between flexion and extension patterns. The fit for the full muscle model $(r=0.146 ; 95 \%$ CI, 0.055-0.235) was as poor as for the kinematic model (twosided paired $t$ test muscle vs kinematic: $t_{(8)}=1.082, p=0.3108$ ) and significantly below the noise ceiling (two-sided paired $t$ test noise ceiling vs muscle: $\left.t_{(8)}=12.701, p=1.39 \mathrm{e}-6\right)$. Thus, neither the co-occurrence of movements nor the pattern of muscle activities can explain the high similarity of activity patterns for finger flexion and extension in M1.

\section{M1 spiking output differs equally for fingers and direction} To what degree is the high similarity between flexion and extension patterns a function of fMRI as the measurement modality? To approach this question, we analyzed the spiking activity of output neurons in M1 during an equivalent single finger individuation task in two trained nonhuman primates ( $M$. mulatta; Schieber and Rivlis, 2005, 2007, data). To facilitate this, we had designed the behavioral task for the human fMRI experiment to closely match the task for the monkeys, such that we could make strong comparisons across species and measurement modalities. Figure $5 \mathrm{~A}$ shows the condition averaged firing rate traces from a single neuron from this dataset. This neuron displayed strong preference (increased firing rates) for flexion of the middle finger and extension of the index finger. As previously reported (Schieber and Hibbard, 1993), the population of M1 neurons demonstrated complex, heterogeneous tuning across fingers and directions.

To compare the representational structure from spiking data to that obtained with fMRI, we calculated the mean firing rate for each neuron from the go-cue onset to the end of the hold phase during each trial. We then calculated dissimilarities between the population responses for different conditions (see Materials and Methods), similar to the analysis of the human EMG and fMRI data. The average RDM is shown in Figure 5C. Similar to the structure of representations in human M1, the thumb activity patterns for both directions were the most distinct, and neighboring fingers had more similar activity patterns. In contrast to the fMRI data, however, the spiking patterns for flexion and extension of the same finger were quite distinct.

To quantify this observation, we averaged dissimilarities between different fingers pressing in the same direction (finger specific) and the same finger pressing in different directions (direction specific). The finger and direction-specific dissimilarities were close in magnitude for both monkeys (Fig. 6A). Also, the human EMG patterns had roughly matched direction- 


\section{A}
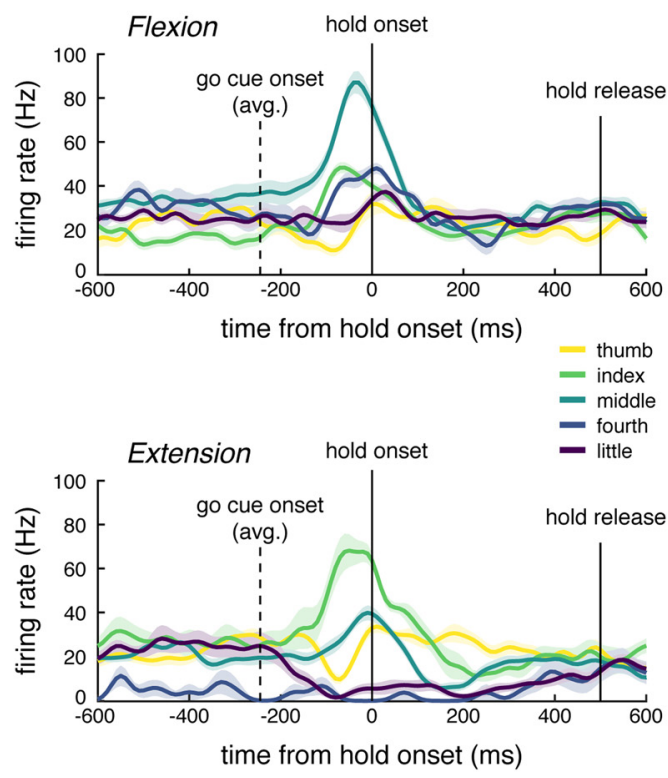

B Activity patterns from Monkey C
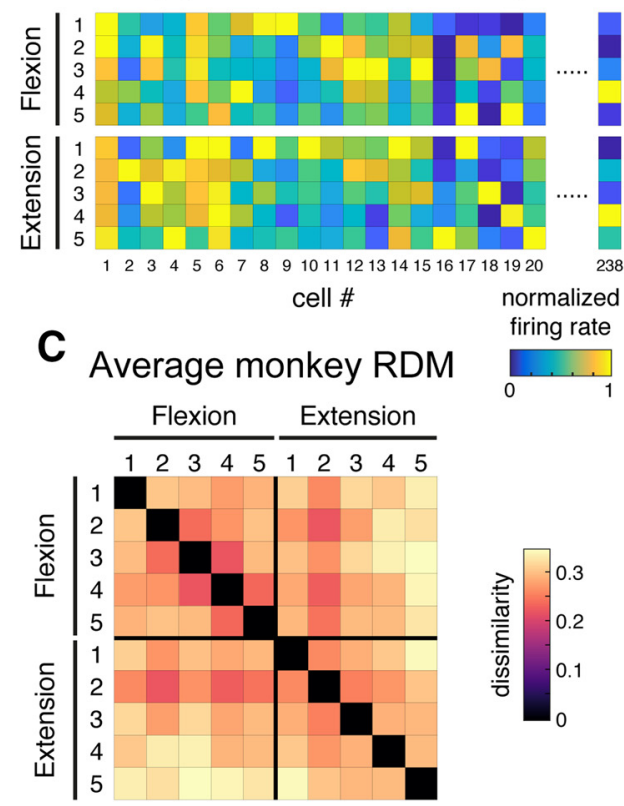

Figure 5. Analysis of M1 spiking activity during monkey single finger flexion and extension. $\boldsymbol{A}$, Trial averaged firing rates from one cell (monkey C). Traces are aligned to press onset ( 0 s). This cell demonstrates selective tuning to middle finger flexion and index finger extension. Firing rates were calculated for $10 \mathrm{~ms}$ bins and smoothed with a Gaussian kernel (FWHM, 50ms). Shaded areas reflect SE across trials. $\boldsymbol{B}$, Averaged firing rates for a subset of cells from monkey $C$, arranged by condition. Cell 13 is plotted in $\boldsymbol{A}$. Firing rates are normalized to the peak rate per cell. C, Average monkey RDM (square root dissimilarities).

specific and finger-specific dissimilarities (Fig. 6B). In contrast, the same analysis on the human fMRI data showed a clear and significant difference between these two kinds of dissimilarities (Fig. 6C).

For a statistical comparison, we then calculated the ratio between dissimilarities between different directions and dissimilarities between different fingers (Fig. 6D). The fMRI ratio was significantly lower than 1 (mean ratio, $0.298 \pm 0.071$; one-sided one-sample $t$ test: $t_{(8)}=-9.858, p=4.72 \mathrm{e}-6$ ), indicating stronger representation of fingers compared with direction. In contrast, both the spiking patterns (monkey $\mathrm{C}$ ratio, 1.173; monkey $\mathrm{G}$ ratio, 1.025) and the human muscle patterns (mean ratio, $0.984 \pm 0.051)$ differed similarly for different fingers and different directions, with the muscle ratios being significantly larger than those for human fMRI (two-sided paired $t$ test: $t_{(8)}=9.733$, $p=1.04 \mathrm{e}-5)$. Thus, we found a clear difference between the structure of fMRI patterns and the structures of spiking and muscle activity patterns.

We suggest that this difference is informative about the general organization of finger flexion and extension movements in M1. The discrepancy between the two measurement modalities can likely be attributed to two (non-mutually exclusive) differences between fMRI and electrophysiology. First, the fMRI signal is dominated by excitatory inputs and local synaptic signaling, and only partly reflects the spiking activity of output neurons (Logothetis et al., 2001). Therefore, the overlapping fMRI activity patterns for flexion and extension might reflect similar inputs and shared local processes within these cortical areas, while the output spiking of these two population remains quite distinct to produce the different patterns of muscle activity required for fine finger control.

Second, fMRI samples a proxy of neuronal activity in a coarse manner, averaging across $\sim 200,000$ cortical neurons $/ \mathrm{mm}^{3}$ in M1 (Young et al., 2013). Thus, even high-resolution fMRI is biased to functional organization at a coarse spatial scale (Kriegeskorte and Diedrichsen, 2016), and so our results could be caused by an organization where neurons tuned to different movement directions for the same finger (or combination of fingers) are clustered together, while neurons that control different fingers or finger combinations are more spatially separated.

\section{Spatial organization of finger and direction related fMRI patterns}

To investigate the second explanation directly, we attempted to determine whether the activity patterns associated with different fingers were organized on a coarser spatial scale than the patterns associated with flexion and extension of a given finger. Using the fMRI data, we calculated to covariance of the finger-specific and direction-specific activations for each pair of voxels within M1 and binned these covariances according to the spatial distance between voxel pairs (see Materials and Methods). If direction is encoded at a finer spatial scale than fingers, we would expect finger effects to be correlated over larger spatial distances.

In contrast to this prediction, the spatial correlation functions for fingers and direction were quite similar (Fig. 6E). We estimated the FWHM of the spatial autocorrelation functions. To account for outliers, we evaluated the median FWHM values. The median FWHM of the finger spatial kernel in M1 was 3.22 $\mathrm{mm}$ (mean $\pm \mathrm{SEM}, 3.44 \pm 0.24 \mathrm{~mm}$ ), comparable to previous reports (Diedrichsen et al., 2011; Wiestler et al., 2011). The median FWHM of the direction spatial kernel in M1 was $4.65 \mathrm{~mm}$ (mean $\pm \mathrm{SEM}, 4.77 \pm 0.84 \mathrm{~mm}$ ), and there was no significant difference between the two (two-sided paired Wilcoxon signedrank test, finger vs direction: $\mathrm{W}=11, p=0.2031$; two-sided paired $t$ test finger vs direction: $t_{(8)}=-1.417, p=0.1942$ ). Therefore, we did not find any direct empirical support for the idea that differences between flexion and extension patterns are organized at a finer spatial scale than differences between fingers. 
However, our analysis was itself limited by the spatial resolution of $7 \mathrm{~T}$ fMRI, such that we cannot rule out the possibility that subpopulations for different directions are interdigitated at a subvoxel scale.

Additionally, we did not find evidence of a substantial spatial separation of flexion versus extension movements, as was suggested by Huber et al. (2020). These authors observed two sets of digit maps in Brodmann area $4 \mathrm{a}$, with one set being more activated for whole hand grasping, and the other more activated for whole-hand retraction movements. From this, the authors suggested that each individual finger map has a preferential function role in guiding flexion and extension movements. To test this idea with our fMRI data, we calculated the CoG of the activity maps for each finger pressing in the flexion and extension directions in Brodmann area 4a (see Materials and Methods).

As shown in Figure 6F, both finger flexion and extension CoGs revealed the expected overall somatotopic gradient, with thumb movements activating more ventrolateral areas and the little finger activating more dorsomedial areas in 4a (two-factor repeatedmeasures MANOVA, finger factor: Wilks' $\left.\Lambda_{(4,32)}=0.28, p=2.2075 \mathrm{e}-6\right)$. However, there was no significant difference in these digit maps across flexion and extension movements (two-factor repeated-measures MANOVA, direction factor: Wilks' $\Lambda_{(1,8)}=0.88, p=0.6427$; finger $\times$ direction interaction: Wilks' $\left.\Lambda_{(4,32)}=0.65, p=0.0793\right)$. We then calculated the pairwise Euclidean distances between the condition CoGs (Fig. 6G) and compared the betweenfinger and within-finger distances, as was done previously. Replicating the results from the fMRI RSA analysis, we found that pressing different fingers resulted in more spatially distinct activation patterns compared with pressing the same finger in different directions (mean ratio, $0.67 \pm 0.04$; one-sided one-sample $t$ test ratio $<1$ : $\left.t_{(8)}=-8.003, p=4.356 \mathrm{e}-5\right)$. This finding is inconsistent with the idea of separate flexion and extension finger maps.

\section{Discussion}

Here we investigated how the population activity in M1 is organized for control of flexion and extension of single fingers. We analyzed M1 population activity measured in humans with 7T fMRI and spiking data from NHPs while participants made

\section{$\mathbf{F}$}
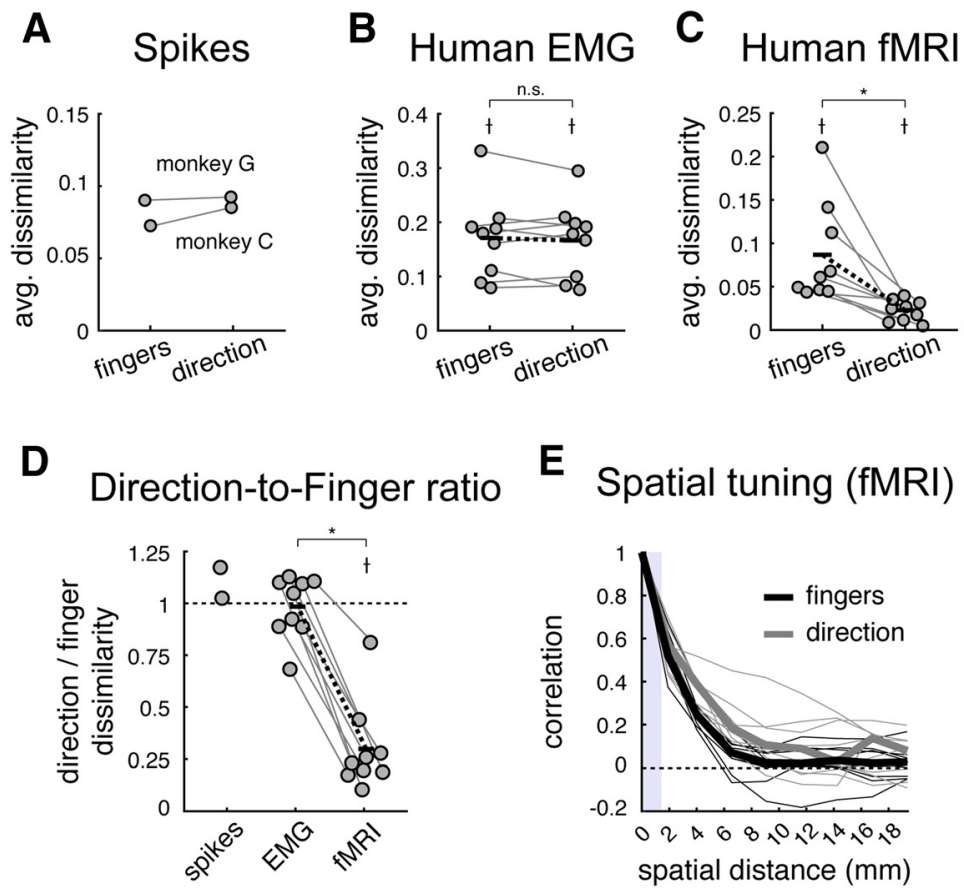

E Spatial tuning (fMRI)
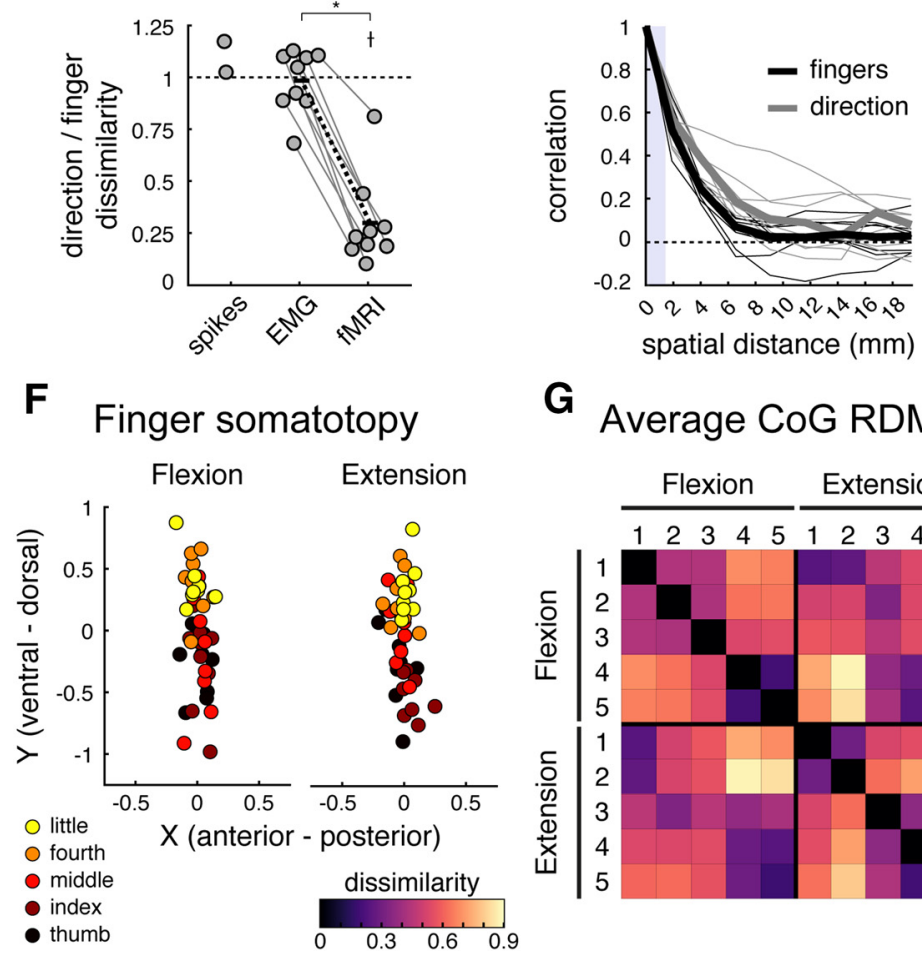

G
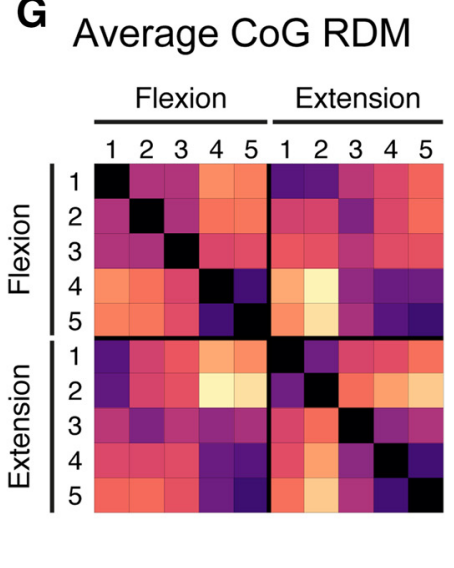

Figure 6. Comparing strength of finger and direction representations across datasets. $A-C$, The average finger- and directionspecific dissimilarities for the spiking $(\boldsymbol{A})$, human EMG $(\boldsymbol{B})$, and human $\mathrm{fMRI}(\boldsymbol{C})$ datasets. Each dot denotes one participant, and lines connect dots from the same participants. Black bars denote the means, and black dashed lines reflect the mean paired differences. 'Dissimilarities significantly larger than zero (one-sided $t$ test, $p<0.05$ ); *significant difference between finger and direction dissimilarities (two-sided paired $t$ test, $p<0.05$ ). $\boldsymbol{D}$, The ratio of the direction-to-finger dissimilarities for each dataset. Values $<1$ indicate stronger finger representation. ${ }^{\dagger}$ Dissimilarities significantly lower than one (one-sided $t$ test, $p<0.05$ ); *significant differences between dissimilarity ratios (two-sided paired $t$ test, $p<0.05$ ); n.s., not significant $(p>0.05)$. $\boldsymbol{E}$, Estimated spatial autocorrelations of finger (black) and direction (gray) pattern components in human M1, plotted as a function of spatial distance between voxels. No significant difference was observed between finger and direction tuning in M1. The thick lines denote the median spatial autocorrelation functions, and small lines are drawn for each participant for each pattern component. The vertical shaded bar denotes the distance between voxel size, for which correlations can be induced by motion correction. $F$, CoG of activation elicited by single finger presses in the flexion or extension direction for each participant. CoGs were aligned across participants before plotting by subtracting the center of the informative region within each participant (i.e., the mean CoG across all conditions). A somatotopic gradient for finger flexion and extension in Brodmann area 4a is visible with the thumb being more ventral, and the little finger more dorsal. $\mathbf{G}$, Group average RDM of the paired Eudlidean distance between condition CoGs.

isometric single finger presses in either direction. Importantly, we ensured that the behavioral tasks in both experiments were carefully matched to allow us to compare results across the two datasets.

We first demonstrated that the representational structure of single finger flexion or extension presses in human M1 measured with fMRI were relatively well explained by the natural statistics of everyday movements, replicating the flexion results reported in the study by Ejaz et al. (2015) and extending them to single 


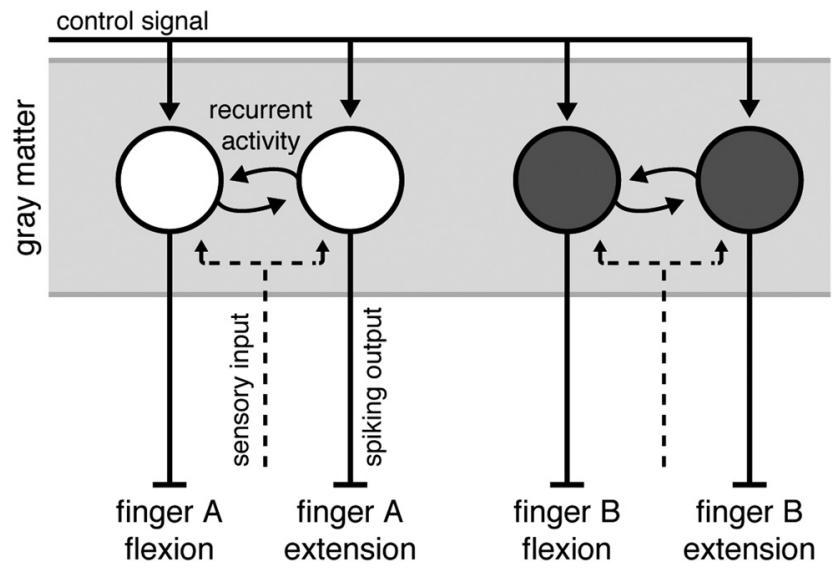

Figure 7. Summary model of M1 organization. Output neurons in M1 produce complex patterns of muscular activity. We refer to groups of neurons that, together, evoke a complex pattern of muscle activity that results in single finger movements as functional units (circles). These functional units receive a control signal input for the upcoming movement (solid lines with arrows). Functional units that evoke movements of the same finger in opposite directions receive common inputs (dashed lines) and share strong recurrent connections (circular lines). The spiking output (solid lines without arrows) of these units, however, is directionally specific. Additionally, under the spatial scale model, functional units tuned to finger movements in different directions are clustered together according to their finger tuning.

finger extension movements. The same model, however, failed to correctly predict the relationship between flexion and extension movements. Because flexion and extension of the same finger cannot temporally co-occur, the model predicted quite separate representations for the two actions. In our data, however, we observed the opposite effect-cortical M1 activity patterns measured with fMRI in humans were very similar for flexion and extension of the same finger, as compared with the quite distinct patterns for different fingers. We also analyzed spiking data from a similar task in two monkeys and found that the similarity of finger flexion and extension was specific to fMRI: in the monkey electrophysiological recordings, different movement directions were associated with distinct patterns of neuronal activity.

The discrepancy between the fMRI and electrophysiological measures suggest a specific organization of finger flexion and extension movements in M1 (Fig. 7). This suggested architecture has two characteristics that likely contribute to the observed difference between measurement modalities.

First, we hypothesize that neurons that contribute to the flexion of a finger receive similar sensory input as neurons that contribute to the extension of the same finger (Fig. 7, dashed line). There is evidence in the literature to support such an organization. In macaque M1, single neurons tuned to torque production at the shoulder integrate information from the shoulder and elbow joints to facilitate rapid corrective responses to mechanical arm perturbations (Pruszynski et al., 2011). Thus, these neurons receive common sensory input about the shoulder and elbow joints, but the output is largely specific to movements about the shoulder. Additionally, units controlling flexion and extension of the same finger are likely to directly communicate with each other (Fig. 7, curved solid arrows). Such coordination would be necessary to orchestrate fast alternation of finger movements and to finely control the grip force during object manipulation.

This organization would lead to highly similar fMRI activity patterns. In cortical gray matter, the BOLD signal measured with fMRI reflects mainly EPSPs, which are caused by input to a region or recurrent activity within a region (Logothetis et al., 2001). This is because much of the metabolic costs associated with signal transmission arise from re-establishing the resting membrane potential of neurons after an EPSP (Attwell and Laughlin, 2001; Magistretti and Allaman, 2015; Yu et al., 2018). Given that the input to subpopulations controlling flexion and extension of the same finger will be highly temporally correlated, the fMRI activity patterns for the two movements should also be very similar.

At the same time, the two subpopulations need to produce distinct spiking outputs. To do so, the populations must receive a control signal input that defines whether to flex or extend a finger. Indeed, in our fMRI data, although flexion and extension patterns for the same finger were highly similar, we could still discriminate between the patterns (Fig. 6C). This control signal would influence how neurons react to sensory inputs and the information they exchange. Thus, the observed local variations in metabolic activity would be dissociated from the local neural firing rates (Picard et al., 2013).

As a second characteristic, we also hypothesize that units controlling muscle patterns that produce flexion and extension of the same effector are spatially colocalized to support fast and efficient communication. Because fMRI samples activity in a coarse manner, even high-resolution fMRI is biased to functional organization at a coarse spatial scale (Kriegeskorte and Diedrichsen, 2016). Therefore, features that exist at fine spatial scales in the neural population are underrepresented in fMRI activity patterns. Our results could therefore be caused by an organization where neurons tuned to different movement directions for the same finger (or combination of fingers) are clustered together, while neurons that control different fingers or finger combinations are more spatially separated. We did not find any evidence for a difference in the spatial organization of fingers and direction in the fMRI data. However, given that this comparison itself is limited by the spatial resolution of fMRI, we cannot rule out that differences in the fine-grained spatial organization also contributed to the observed effect.

Although we experimentally studied the flexion and extension of single fingers, we do not suggest that isolated finger movements are explicitly represented in M1. Rather, M1 output neurons will produce a complex pattern of muscle activity. This complexity likely arises because the neuronal populations are optimized to produce muscle activities that elicit combinations of finger movements that are useful in everyday tasks (Poliakov and Schieber, 1999; Gentner and Classen, 2006; Ejaz et al., 2015). When we measure activity patterns related to movements of isolated fingers, we simply observe the specific combination of neuronal populations that need to be active to move a single finger (Schieber, 1990). The core of our hypothesis is that populations of neurons that produce opposing muscular patterns form a functional unit with increased communication, common sensory input, and potentially also spatial colocalization.

Our findings are at odds with the organization suggested by Huber et al. (2020). Using high-resolution functional imaging in humans, the authors reported evidence of two spatially distinct finger maps in M1: one for flexion and one for extension. Consistent with Huber et al. (2020), we found that individuated finger activity patterns in M1 are fractured and have multiple hotspots (Fig. 2). However, we found no evidence for a clear spatial separation of finger flexion and extension into two action maps (Fig. 6F,G). Although the spatial resolution of BOLD imaging in our study was lower than that of the blood volume-based method used by Huber et al. (2020), we should have been able to detect larger spatial separations between flexion and extension movements than between individual fingers. Instead, the 
opposite was the case. Both the RSA and the spatial analyses showed greater differences between fingers than between directions. These results, however, are not unexpected. Partial inactivation of neurons in the hand area of macaque M1 result in a complex loss of flexion and/or extension movements of different fingers (Schieber and Poliakov, 1998), and electrophysiological recordings from this same area show that flexion and extension preference is not spatially clustered (Schieber and Hibbard, 1993). We believe that the differences between our results and those of Huber et al. (2020) are likely explained by the fact that Huber et al. (2020) did not study flexion and extension of individual fingers, but relied on a large spatial gradient detected between whole-hand grasping and retraction. We think this is problematic, as the control requirements of individual finger movements is qualitatively different from those of whole-hand grasping. That is, neuronal activity during whole-hand grasping is not the sum of the neural activity during individuated finger flexion movements (Ejaz et al., 2015) but, rather, engages a different control mechanism. Consistent with this idea, electrophysiological studies have shown that the neural control of wholehand and individuated finger movements relies on different neural subpopulations (Muir and Lemon, 1983; Lemon, 2008).

There are of course many caveats when comparing results across different recording methodologies, experimental setups, and species. While we tried to make the behavioral tasks across human and macaques as similar as possible, species differences or the extensive training for the nonhuman primates may account for some of the differences.

Overall, however, we believe that the comparison between fMRI and spiking provides some interesting insights into the organization of the hand region of the primary motor cortex. Cortical representations of single finger movements are not purely dictated by the kinematics of hand usage. We posit that the deviation from this organization appears to reflect a control process, where neurons tuned to movements of a specific finger receive common sensory input and share local recurrent processes. These tightly coordinated populations then produce the spiking output that needs to be quite distinct for the flexion and extension of the same finger.

\section{References}

Arbuckle SA, Yokoi A, Pruszynski JA, Diedrichsen J (2019) Stability of representational geometry across a wide range of fMRI activity levels. Neuroimage 186:155-163.

Attwell D, Laughlin SB (2001) An energy budget for signaling in the grey matter of the brain. J Cereb Blood Flow Metab 21:1133-1145.

Beringer CR, Mansouri M, Fisher LE, Collinger JL, Munin MC, Boninger ML, Gaunt RA (2020) The effect of wrist posture on extrinsic finger muscle activity during single joint movements. Sci Rep 10:8377.

Bortoff GA, Strick PL (1993) Corticospinal terminations in two new-world primates: further evidence that corticomotoneuronal connections provide part of the neural substrate for manual dexterity. J Neurosci 13:51055118.

Darling WG, Pizzimenti MA, Rotella DL, Peterson CR, Hynes SM, Ge J, Solon K, McNeal DW, Stilwell-Morecraft KS, Morecraft RJ (2009) Volumetric effects of motor cortex injury on recovery of dexterous movements. Exp Neurol 220:90-108.

Diedrichsen J, Ridgway GR, Friston KJ, Wiestler T (2011) Comparing the similarity and spatial structure of neural representations: a pattern-component model. Neuroimage 55:1665-1678.

Diedrichsen J, Provost S, Zareamoghaddam H (2016) On the distribution of cross-validated Mahalanobis distances. arXiv. Advance online publication. Retrieved October 22, 2020. arXiv:1607.01371v1.

Diedrichsen J, Yokoi A, Arbuckle SA (2018) Pattern component modeling: a flexible approach for understanding the representational structure of brain activity patterns. Neuroimage 180:119-133.
Diedrichsen J, Berlot E, Mur M, Schütt HH, Kriegeskorte N (2020) Comparing representational geometries using the unbiased distance correlation. arXiv. Advance online publication. Retrieved October 22, 2020. arXiv:2007.02789.

Ejaz N, Hamada M, Diedrichsen J (2015) Hand use predicts the structure of representations in sensorimotor cortex. Nat Neurosci 18:1034-1040.

Fischl B, Sereno MI, Dale AM (1999a) Cortical surface-based analysis. II: inflation, flattening, and a surface-based coordinate system. Neuroimage 9:195-207.

Fischl B, Sereno MI, Tootell RBH, Dale AM (1999b) High-resolution intersubject averaging and a coordinate system for the cortical surface. Hum Brain Mapp 8:272-284.

Fischl B, Rajendran N, Busa E, Augustinack J, Hinds O, Yeo BTT, Mohlberg $\mathrm{H}$, Amunts K, Zilles K (2008) Cortical folding patterns and predicting cytoarchitecture. Cereb Cortex 18:1973-1980.

Friston KJ, Jezzard P, Turner R (1994) Analysis of functional MRI time-series. Hum Brain Mapp 1:153-171.

Gentner R, Classen J (2006) Modular organization of finger movements by the human central nervous system. Neuron 52:731-742.

Graziano MS, Aflalo TN (2007) Mapping behavioral repertoire onto the cortex. Neuron 56:239-251.

Heffner RS, Masterton RB (1983) The role of the corticospinal tract in the evolution of human digital dexterity. Brain Behav Evol 23:165-183.

Huber L, Finn ES, Handwerker DA, Bönstrup M, Glen DR, Kashyap S, Ivanov D, Petridou N, Marrett S, Goense J, Poser BA, Bandettini PA (2020) Sub-millimeter fMRI reveals multiple topographical digit representations that form action maps in human motor cortex. Neuroimage 208:116463.

Hutton C, Bork A, Josephs O, Deichmann R, Ashburner J, Turner R (2002) Image distortion correction in fMRI: a quantitative evaluation. Neuroimage 16:217-240.

Ingram JN, Körding KP, Howard IS, Wolpert DM (2008) The statistics of natural hand movements. Exp Brain Res 188:223-236.

Kriegeskorte N, Diedrichsen J (2016) Inferring brain-computational mechanisms with models of activity measurements. Philos Trans R Soc Lond B Biol Sci 371:20160278.

Lang CE, Schieber MH (2003) Differential impairment of individuated finger movements in humans after damage to the motor cortex or the corticospinal tract. J Neurophysiol 90:1160-1170.

Lawrence DG, Hopkins DA (1976) The development of motor control in the rhesus monkey: evidence concerning the role of corticomotoneuronal connections. Brain 99:235-254.

Lawrence DG, Kuypers HG (1968) The functional organization of the motor system in the monkey. I. The effects of bilateral pyramidal lesions. Brain 91:1-14.

Lemon RN (2008) Descending pathways in motor control. Annu Rev Neurosci 31:195-218.

Li Z-M, Pfaeffle HJ, Sotereanos DG, Goitz RJ, Woo SL-Y (2003) Multi-directional strength and force envelope of the index finger. Clin Biomech (Bristol, Avon) 18:908-915.

Liu Y, Rouiller EM (1999) Mechanisms of recovery of dexterity following unilateral lesion of the sensorimotor cortex in adult monkeys. Exp Brain Res 128:149-159.

Logothetis NK, Pauls J, Augath M, Trinath T, Oeltermann A (2001) Neurophysiological investigation of the basis of the fMRI signal. Nature 412:150-157.

Magistretti PJ, Allaman I (2015) A cellular perspective on brain energy metabolism and functional imaging. Neuron 86:883-901.

Mogk JP, Keir PJ (2003) The effects of posture on forearm muscle loading during gripping. Ergonomics 46:956-975.

Muir RB, Lemon RN (1983) Corticospinal neurons with a special role in precision grip. Brain Res 261:312-316.

Nili H, Wingfield C, Walther A, Su L, Marslen-Wilson W, Kriegeskorte N (2014) A toolbox for representational similarity analysis. PLoS Comput Biol 10:e1003553.

Picard N, Matsuzaka Y, Strick PL (2013) Extended practice of a motor skill is associated with reduced metabolic activity in M1. Nat Neurosci 16:13401347.

Poliakov AV, Schieber MH (1999) Limited functional grouping of neurons in the motor cortex hand area during individuated finger movements: a cluster analysis. J Neurophysiol 82:3488-3505. 
Pruszynski JA, Kurtzer I, Nashed JY, Omrani M, Brouwer B, Scott SH (2011) Primary motor cortex underlies multi-joint integration for fast feedback control. Nature 478:387-390.

Sasaki S, Isa T, Pettersson L-G, Alstermark B, Naito K, Yoshimura K, Seki K, Ohki Y (2004) Dexterous finger movements in primate without monosynaptic corticomotoneuronal excitation. J Neurophysiol 92:3142-3147.

Schieber MH (1990) How might the motor cortex individuate movements? Trends Neurosci 13:440-445.

Schieber MH (1991) Individuated finger movements of rhesus monkeys: a means of quantifying the independence of the digits. J Neurophysiol 65:1381-1391.

Schieber MH, Hibbard LS (1993) How somatotopic is the motor cortex hand area? Science 261:489-492.

Schieber MH, Poliakov AV (1998) Partial inactivation of the primary motor cortex hand area: effects on individuated finger movements. J Neurosci 18:9038-9054

Schieber MH, Rivlis G (2005) A spectrum from pure post-spike effects to synchrony effects in spike-triggered averages of electromyographic activity during skilled finger movements. J Neurophysiol 94:3325-3341.

Schieber MH, Rivlis G (2007) Partial reconstruction of muscle activity from a pruned network of diverse motor cortex neurons. J Neurophysiol 97:70-82.

Tower SS (1940) Pyramidal lesion in the monkey. Brain 63:36-90.

Valero-Cuevas FJ, Zajac FE, Burgar CG (1998) Large index-fingertip forces are produced by subject-independent patterns of muscle excitation. J. Biomech 31:693-703.
Walther A, Nili H, Ejaz N, Alink A, Kriegeskorte N, Diedrichsen J (2016) Reliability of dissimilarity measures for multi-voxel pattern analysis. Neuroimage 137:188-200.

Wiestler T, McGonigle DJ, Diedrichsen J (2011) Integration of sensory and motor representations of single fingers in the human cerebellum. J Neurophysiol 105:3042-3053.

Xu J, Ejaz N, Hertler B, Branscheidt M, Widmer M, Faria AV, Harran MD, Cortes JC, Kim N, Celnik PA, Kitago T, Luft AR, Krakauer JW, Diedrichsen J (2017) Separable systems for recovery of finger strength and control after stroke. J Neurophysiol 118:1151-1163.

Young NA, Collins CE, Kaas JH (2013) Cell and neuron densities in the primary motor cortex of primates. Front Neural Circuits 7:30.

Yousry TA, Schmid UD, Alkadhi H, Schmidt D, Peraud A, Buettner A, Winkler P (1997) Localization of the motor hand area to a knob on the precentral gyrus. A new landmark. Brain 120:141-157.

Yu BM, Cunningham JP, Santhanam G, Ryu SI, Shenoy KV, Sahani M (2009) Gaussian-process factor analysis for low-dimensional singletrial analysis of neural population activity. J Neurophysiol 102:614635.

Yu WS,Duinen H, Gandevia SC (2010) Limits to the control of the human thumb and fingers in flexion and extension. J Neurophysiol $103: 278-289$

Yu Y, Herman P, Rothman DL, Agarwal D, Hyder F (2018) Evaluating the gray and white matter energy budgets of human brain function. J Cereb Blood Flow Metab 38:1339-1353. 